\title{
SIMPLE RESONANCE IN NONLINEAR TORSIONAL VIBRATION SYSTEMS OF THREE VARIABLE GENERALIZED MASSES
}

\author{
NguYen Van Khang - Tran Dinh Son \\ Hanoi Technical University, Vietnam
}

\section{Introduction}

Torsional vibrations of transmission systems are often encountered in mechanical engineering. Torsional vibration analysis of linear systems has drawn the attention of investigators [1-4]. However, such an attention to nonlinear systems has not been paid properly.

In this paper our objective is to examine simple resonance solutions for a torsional vibration system of three variable generalized masses by using the small parameter method [5].

\section{Differential equations of vibration}

Let us consider a nonlinear torsional vibration system of three variable generalized masses as shown in Fig. 1

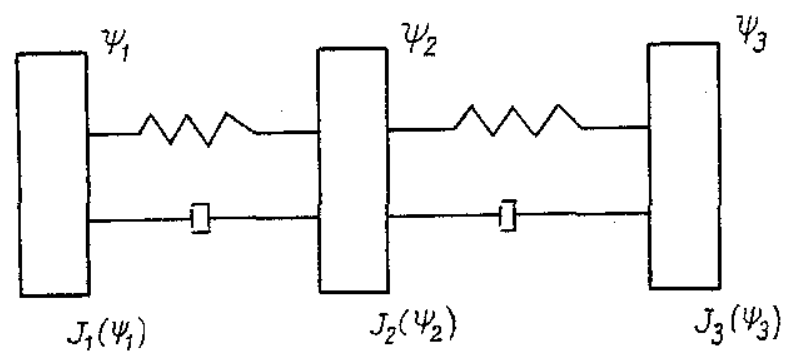

Fig. 1 that

In technical practice, it is often the case that $J_{1}=$ const while $J_{2}$ and $J_{3}$ depend on $\psi_{i}$ so

$$
J_{i}=J_{0 i}+J_{1 i} \cos 2 \psi_{i}
$$

where $J_{0 i}$ and $J_{1 i}$ are constants.

For more explicitness, we consider the case when $\dot{\psi}_{1}=\Omega=$ const and the angles $\psi_{2}$ and $\psi_{3}$ can be written in the form

$$
\psi_{i}=\Omega t+\varphi_{i}, \quad(i=2,3)
$$

Further, it is assumed that the damping coefficients $b_{i}^{(1)}, b_{i}^{(3)}$ and nonlinearly elastic coefficients 
$c_{i}^{(3)}$ are small, while reduced moments of inertia are written in the form:

$$
J_{i}=J_{0 i}\left(1+\frac{J_{1 i}}{J_{0 i}} \cos 2 \psi_{i}\right)=J_{0 i}\left(1+\varepsilon \bar{J}_{1 i} \cos 2 \psi_{i}\right), \quad(i=2,3),
$$

where $\varepsilon$ is a small parameter.

Taking into consideration all assumptions; the vibration differential equation of the system is given under the form $[6,8]$

$$
\underline{M} \ddot{\vec{q}}+\underline{C} \vec{q}=\epsilon \vec{\phi}
$$

where

$$
\underline{M}=\left[\begin{array}{cc}
J_{02} & 0 \\
0 & J_{03}
\end{array}\right], \quad \vec{q}=\left[\begin{array}{l}
\varphi_{2} \\
\varphi_{3}
\end{array}\right], \quad \underline{C}=\left[\begin{array}{cc}
c_{1}^{(1)}+c_{2}^{(1)} & -c_{2}^{(1)} \\
-c_{2}^{(1)} & c_{2}^{(1)}
\end{array}\right], \quad \vec{\phi}=\left[\begin{array}{l}
\phi_{2} \\
\phi_{3}
\end{array}\right] .
$$

The functions $\phi_{2}, \phi_{3}$ have the following expressions [8]

$$
\begin{aligned}
\phi_{2}= & -\frac{1}{2} \Omega^{2} \bar{J}_{2,2}-\bar{J}_{2,2}\left(\varphi_{2} \ddot{\varphi}_{2}+\frac{1}{2} \dot{\varphi}_{2}^{2}\right)-\bar{J}_{2,22}\left(\frac{1}{2} \varphi_{2}^{2} \ddot{\varphi}_{2}+\frac{1}{2} \varphi_{2} \dot{\varphi}_{2}^{2}+\Omega \varphi_{2} \dot{\varphi}_{2}\right) \\
& -\bar{J}_{2,222}\left(\frac{1}{2} \Omega \varphi_{2}^{2} \dot{\varphi}_{2}+\frac{1}{4} \Omega^{2} \varphi_{2}^{2}\right)-\frac{1}{12} \Omega^{2} \bar{J}_{2,2222} \varphi_{2}^{3}-c_{1}^{(3)} \varphi_{2}^{3} \\
& -c_{2}^{(3)}\left(\varphi_{2}-\varphi_{3}\right)^{3}-b_{1}^{(3)} \dot{\varphi}_{2}^{3}-b_{2}^{(3)}\left(\dot{\varphi}_{2}-\dot{\varphi}_{3}\right)^{3}-b_{1}^{(1)} \dot{\varphi}_{2} \\
& -b_{2}^{(1)}\left(\dot{\varphi}_{2}-\dot{\varphi}_{3}\right)-\Omega \bar{J}_{2,2} \dot{\varphi}_{2}-\frac{1}{2} \Omega^{2} \bar{J}_{2,22} \varphi_{2}, \\
\phi_{3}= & -\frac{1}{2} \Omega^{2} \bar{J}_{3,3}-\bar{J}_{3,3}\left(\varphi_{3} \ddot{\varphi}_{3}+\frac{1}{2} \dot{\varphi}_{3}^{2}\right)-\bar{\varphi}_{3,33}\left(\frac{1}{2} \varphi_{3}^{2} \ddot{\varphi}_{3}+\frac{1}{2} \varphi_{3} \dot{\varphi}_{3}^{2}+\Omega \varphi_{3} \dot{\varphi}_{3}\right) \\
& -\bar{J}_{3,333}\left(\frac{1}{2} \Omega \varphi_{3}^{2} \dot{\varphi}_{3}+\frac{1}{4} \Omega^{2} \varphi_{3}^{2}\right)-\frac{1}{12} \Omega^{2} \bar{J}_{3,3333} \varphi_{3}^{3}+c_{2}^{(3)}\left(\varphi_{2}-\varphi_{3}\right)^{3} \\
& +b_{2}^{(3)}\left(\dot{\varphi}_{2}-\dot{\varphi}_{3}\right)^{3}+b_{2}^{(1)}\left(\dot{\varphi}_{2}-\dot{\varphi}_{3}\right)-\Omega \bar{J}_{3,3} \dot{\varphi}_{3}-\frac{1}{2} \Omega^{2} \bar{J}_{3,33} \varphi_{3} .
\end{aligned}
$$

In order to have an equation in modal coordinates we make a coordinate transformation relating physical coordinates $\vec{q}$ to the modal ones $\vec{p}$

$$
\vec{q}=\underline{V} \vec{p}
$$

in which $\underline{V}$ is the modal matrix. The matrix $V$ has the form $[8]$

$$
V=\left[\begin{array}{cc}
1 & 1 \\
v_{2}^{(1)} & v_{2}^{(2)}
\end{array}\right]=\left[\begin{array}{cc}
1 & 1 \\
\frac{c_{1}^{(1)}+c_{2}^{(1)}-J_{02} \omega_{1}^{2}}{c_{2}^{(1)}} & \frac{c_{1}^{(1)}+c_{2}^{(1)}-J_{02} \omega_{2}^{2}}{c_{2}^{(1)}}
\end{array}\right]
$$

where

$$
\omega_{1,2}^{2}=\frac{c_{2}^{(1)} J_{02}+J_{03}\left(c_{1}^{(1)}+c_{2}^{(1)}\right) \pm \sqrt{\left[c_{2}^{(1)} J_{02}+J_{03}\left(c_{1}^{(1)}+c_{2}^{(1)}\right)\right]^{2}-4 c_{1}^{(1)} c_{2}^{(1)} J_{02} J_{03}}}{2 J_{02} J_{03}} .
$$

Substituting the expression (2.5) into equation (2.2), we obtain the vibration differential equations in the modal coordinates

$$
p_{i}^{\prime \prime}+\lambda_{i} p_{i}=\varepsilon F_{i}
$$


in which

$$
\begin{aligned}
F_{i}= & \sum_{j} a_{i j} \cos 2 m \tau p_{j}+\sum_{j}\left(\bar{a}_{i j} \sin 2 m \tau+\bar{a}_{i j}^{0}\right) p_{j}^{\prime}+\sum_{j, k} b_{i j k} \sin 2 m \tau p_{j} p_{k} \\
& +\sum_{j, k} \bar{b}_{i j k} \sin 2 m \tau p_{j}^{\prime} \bar{p}_{k}^{\prime}+\sum_{j, k} \bar{c}_{i j k} \sin 2 m \tau p_{j} p_{k}^{\prime \prime}+\sum_{j, k} c_{i j k} \cos 2 m \tau p_{j} p_{k} \\
& +\sum_{j, k, \ell}\left(d_{i j k \ell} \cos 2 m \tau+d_{i j k \ell}^{0}\right) p_{j} p_{k} p_{\ell}+\sum_{j, k, \ell} \bar{d}_{i j k \ell}^{0} p_{j}^{\prime} p_{k}^{\prime} p_{\ell}^{\prime} \\
& +\sum_{j, k, \ell}\left(e_{i j k \ell} \sin 2 m \tau p_{j} p_{k} p_{\ell}^{\prime}\right)+\sum_{j, k, \ell} \bar{e}_{i j k \ell} \cos 2 m \tau p_{j} p_{k} p_{\ell}^{\prime \prime} \\
& +\sum_{j, k, \ell} f_{i j k \ell} \cos 2 m \tau p_{j} p_{k}^{\prime} p_{\ell}^{\prime}+a_{i} \sin 2 m \tau .
\end{aligned}
$$

The indices $j, k, \ell$ are taken for the values 1 and 2 and the coefficients in (2.8) are determined in [8].

3. Analysis of simple resonance

It is supposed that

$$
\lambda_{1}=n^{2}(1-\varepsilon \alpha),
$$

where $n$ is an integer. By introducing the notations:

$$
\Lambda_{1}=n^{2}, \quad \Lambda_{2}=\lambda_{2},
$$

the equation (2.7) can be rewritten under the form:

$$
p_{i}^{\prime \prime}+\Lambda_{i} p_{i}=\varepsilon\left(F_{i}+n^{2} \alpha \delta_{i}^{1} p_{i}\right) .
$$

In the first approximation we shall find the solution of (3.1) in the form:

$$
p_{i}^{(0)}=\delta_{i}^{1}(R \cos n \tau+S \sin n \tau) .
$$

According to [5] the condition for the existence of periodic solutions of period $2 \pi$ implies that:

$$
\int_{0}^{2 \pi}\left(F_{1}+n^{2} p_{1}^{(0)}\right)\left(\begin{array}{c}
\cos n \tau \\
\sin n \tau
\end{array}\right) d \tau=0
$$

From this we obtain the amplitude-frequency equation as follows: 


$$
\begin{aligned}
& \left(n^{2} \alpha+\frac{3 d_{111}^{0} A^{2}}{4}\right)\left(\begin{array}{c}
R \\
S
\end{array}\right)+\frac{a_{11}-n \bar{a}_{11}}{2}\left(\begin{array}{c}
R \\
-S
\end{array}\right) \delta_{m}^{n}+\frac{d_{1111}}{2}\left(\begin{array}{c}
R^{3} \\
-S^{3}
\end{array}\right) \delta_{m}^{n}+ \\
& \frac{n^{2}}{2} \bar{e}_{111}\left(\begin{array}{c}
-R^{3} \\
S^{3}
\end{array}\right) \delta_{m}^{n}+\frac{n e_{1111}}{4}\left(\begin{array}{c}
-R^{3}+R S^{2} \\
S^{3}-S R^{2}
\end{array}\right) \delta_{m}^{n}+\frac{n^{2}}{2}\left(\begin{array}{c}
R S^{2} \\
-S R^{2}
\end{array}\right) f_{1111} \delta_{m}^{n}+ \\
& a_{1}\left(\begin{array}{c}
0 \\
1
\end{array}\right) \delta_{m}^{n / 2}+\frac{b_{111}}{4}\left(\begin{array}{c}
2 R S \\
A^{2}+2 S^{2}
\end{array}\right) \delta_{m}^{n / 2}+\frac{n^{2} \bar{b}_{111}}{4}\left(\begin{array}{c}
-2 R S \\
3 R^{2}+S^{2}
\end{array}\right) \delta_{m}^{n / 2}+ \\
& \frac{n c_{111}}{4}\left(\begin{array}{c}
2 R S \\
S^{2}-R^{2}
\end{array}\right) \delta_{m}^{n / 2}-\frac{n^{2} \bar{c}_{111}}{4}\left(\begin{array}{c}
2 R S \\
3 S^{2}+R^{2}
\end{array}\right) \delta_{m}^{n / 2}+ \\
& \frac{b_{111}}{4}\left(\begin{array}{c}
2 R S \\
R^{2}-S^{2}
\end{array}\right) \delta_{m}^{3 n / 2}+\frac{n^{2} \bar{b}_{111}}{4}\left(\begin{array}{c}
-2 R S \\
-R^{2}+S^{2}
\end{array}\right) \delta_{m}^{3 n / 2}+ \\
& \frac{n c_{111}}{4}\left(\begin{array}{c}
2 R S \\
R^{2}-S^{2}
\end{array}\right) \delta_{m}^{3 n / 2}-\frac{n^{2} \bar{c}_{111}}{4}\left(\begin{array}{c}
2 R S \\
R^{2}-S^{2}
\end{array}\right) \delta_{m}^{3 n / 2}+ \\
& \frac{d_{1111}-n e_{1111}-n^{2} \bar{e}_{1111}}{8}\left(\begin{array}{c}
R^{3}-3 R S^{2} \\
S^{3}-3 S R^{2}
\end{array}\right) \delta_{m}^{2 n}-\frac{n^{2} f_{1111}}{8} A^{2}\left(\begin{array}{c}
R \\
S
\end{array}\right) \delta_{m}^{2 n}+ \\
& n \bar{a}_{11}^{0}\left(\begin{array}{c}
S \\
-R
\end{array}\right)+\frac{3 n^{3} \bar{d}_{1111}^{0} A^{2}}{4}\left(\begin{array}{c}
S \\
-R
\end{array}\right)=\left(\begin{array}{l}
0 \\
0
\end{array}\right) .
\end{aligned}
$$

The equation (3.4) shows that the following resonance cases might occur:

- Fundamental resonance: $\omega_{2}=\Omega,(m=n)$.

- Superharmonic resonance: $\omega_{2}=\frac{\omega}{2},(m=2 n)$.

- Super-subharmonic resonance: $\omega_{2}=\frac{2 \Omega}{3},\left(m=\frac{3 n}{2}\right)$.

- Subharmonic resonance of $2^{\text {nd }}$ - kind: $\omega_{2}=2 \Omega,\left(m=\frac{n}{2}\right)$

Consider now the super-subharmonic resonance for $\omega_{2}=\frac{2 \Omega}{3}$. The equation (3.4) yield

$$
n^{2} \alpha=-\frac{3 f A^{2}}{4} \pm \sqrt{\frac{c^{2} A^{2}}{144}-\left(n b+\frac{3 n^{3} g A^{2}}{4}\right)^{2}}
$$

in which

$$
c=b_{111} ; \quad b=\bar{a}_{11}^{0} ; \quad a=a_{11} ; \quad e=d_{1111} ; \quad f=d_{1111}^{0} ; \quad g=\bar{d}_{1111}^{0} ; \quad d=c_{111} .
$$

We can find the stability condition for this case with the help of the condition established in [5].

If the positive sign or the negative sign is choosen before the square root, the stability condition will be respectively:

$$
\frac{d \alpha}{d A}<0 \text { or } \frac{d \alpha}{d A}>0
$$

For the solution $A=0$, it becomes

$$
b<0, \quad n^{2} b^{2}>-n^{2} \alpha^{2} .
$$

From here we conclude that the solution $A=0$ is always stable. 
After similar calculations for other cases we obtain the results presented in the table below

\begin{tabular}{lc}
\hline $\begin{array}{c}\text { Resonance Case } \\
\omega_{2}=\Omega(m=n)\end{array}$ & Expression of Amplitude \\
$\omega_{2}=\frac{2 \Omega}{3}\left(m=\frac{3 n}{2}\right)$ & $n^{2} \alpha=-\frac{3 f A^{3}}{4} \pm \frac{2 a+e A^{2}}{2\left(4 a+e A^{2}\right)} \sqrt{\left(4 a+e A^{2}\right)^{2}-\left(4 n b+3 n^{3} g A^{2}\right)^{2}}$ \\
$\omega_{2}=2 \Omega\left(m=\frac{n}{\frac{a^{2} A^{2}}{4}-\left(n b+\frac{3 n^{3} g A^{2}}{4}\right)^{2}}\right.$ \\
$\omega_{2}=\frac{\Omega}{2}(m=2 n)$ & $n^{2} \alpha=-\frac{3 f A^{2}}{4}$ \\
\hline
\end{tabular}

In Fig. 2 the amplitude - frequence curve is plotted for the case $m=3 n / 2$. The heavy line corresponds to the stable vibration and the dashed line - to the unstable one.

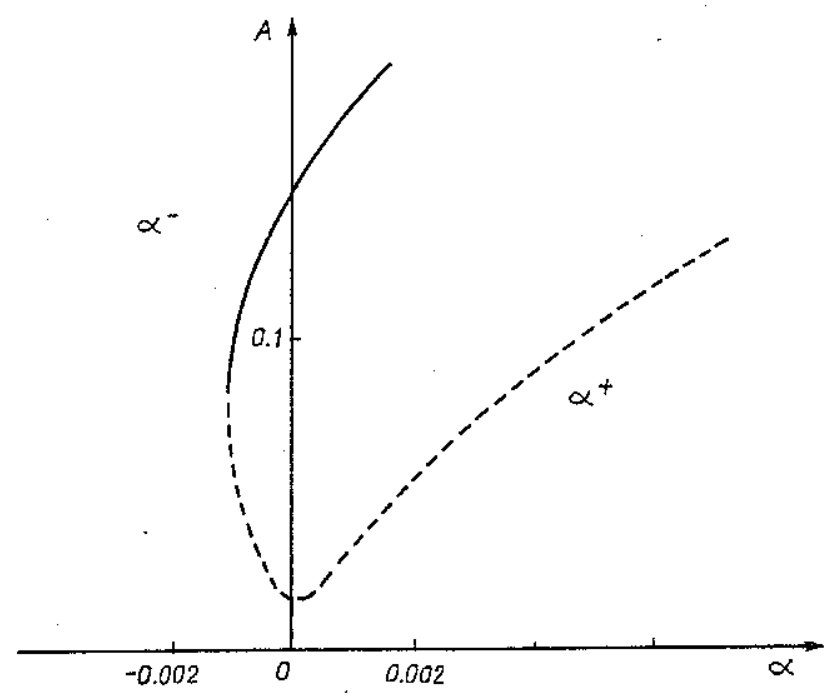

Fig. 2

In Fig. 3 the result from computer simulation is presented for $\alpha=-10^{3}$. Comparing this result with the one obtained in Fig. 2: $A \approx 0.087$ for $\alpha=-10^{-3}$, it is seen that these results are coincident. 


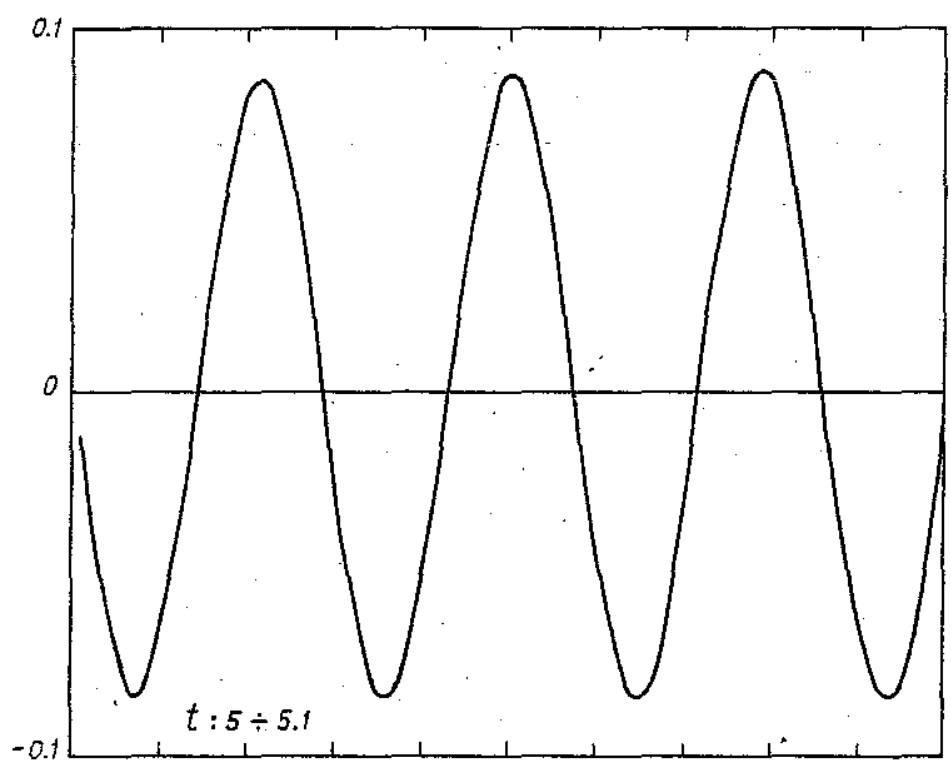

Fig. 9

\section{Conclusion}

The calculation of nonlinear parametrical torsional vibration presents a quite complicated problem. In this paper an amplitude-frequency equation for a nonlinear torsional vibration system of three variable generalized masses was set up. The result of analytical investigation perfectly coincides with the ones obtained from computer simulation. The combined resonance vibration will be given in an other publication.

This work is completed with financial support from the National Basic Research Programme in Natural Sciences.

\section{References}

1. Hafner K. E., Mass H. Torsionsschwingungen in der Verbrennungskraftmas chine. Springer Verlag, Wien 1965.

2. Hokzweissig F., Dresig H. Lehrbuch der Maschinendynamik. (3. Auflage). Fachbuchverlage, Leipzig-Köln 1992.

3. Ho Tan Chuan, Nguyen Duc Phu, Tran Vam Te, Nguyen Tat Lien. Construction and calculation of internal - combustion engine, (in Vietmamese). University and Secondary vocational School Publishing House, Hanoi 1984.

4. Phan Nguyen Di, Nguyen Van Khang. Vibration calculation of machines (in Vietnamese). Science and Technics Publishing House, Hanoi 1991.

5. Nguyen Van Khang. Determination of the oscillations in the systems with nonlinear inertia 
term by the method of small parameter (in Vietnamese). Vietnamese Journal of Mechanics, No 4, p.12-18, Hanoi 1980.

6. Nguyen Van Khang, Tran Dinh Son. On a model of the nonlinear torsional vibration with variable generalized masses (in Vietnamese). Vietnamese Journal of Mechanics, No 2, p. 17-22, Hanoi 1992.

7. Nguyen Van Khang, Tran Dinh Son. On the nonlinear parametric resonance of a torsional vibration system with variable generalized masses (in Vietnamese). Vietnamese Journal of Mechanics, No 4, p. 10-18, Hanoi 1992.

8. Tran Dinh Son. Torsional vibration calculation of system with variable generalized masses (in Vietnamese). Doctor's thesis, Hanoi 1996.

Received March 2, 1997

\section{VỀ CÁC CộNG HỨ̛̛NG ĐON CỬA HỆ DAO ĐộNG XOÁN PHI TUYẾN CÓ BA KHỐI LƯợ̂G THU GỌN BIẾN ĐỔI}

Trong bài báo này đã thiết lập được phương trình biên độ - tần số cho một hệ dao động xoån phi tuyến có ba khối lượng thu gọn biến đổi. Các kết quả thu được bằng phương pháp giải tích phù hợp với các kết quả thu được bằng mô phơng số. 